Original Research Article

\title{
Comparison of protective effects of carvedilol and $\alpha$-tocopherol on doxorubicin-induced cardiotoxocity
}

\author{
Khalida Ajmal $^{1 *}$, Mohammad Ajmal ${ }^{2}$, Ayesha Afzal ${ }^{1}$, Saima $^{1}$, Uzma Naeem ${ }^{3}$, Abeera Sikandar
}

Department of Pharmacology,

${ }^{1}$ Wah Medical College, Wah

Cantt, Rawalpindi, ${ }^{3}$ Islamic

International Medical College,

Islamabad, Pakistan

${ }^{2}$ Department of Radiology,

POFs Hospital, Wah Cantt,

Pakistan

Received: 14 May 2019

Revised: 13 June 2019

Accepted: 31 July 2019

*Correspondence to:

Dr. Khalida Ajmal,

Email: mrskhalida57@

gmail.com

Copyright: (C) the author(s), publisher and licensee Medip Academy. This is an openaccess article distributed under the terms of the Creative Commons Attribution NonCommercial License, which permits unrestricted noncommercial use, distribution, and reproduction in any medium, provided the original work is properly cited.

\begin{abstract}
Background: Doxorubicin, an effective anticancer drug used to treat multiple solid tumours and childhood malignancies since many decades but its cardiac adverse effects limits its use in full therapeutic dose. The mechanism involved in cardiotoxicity is apoptosis of cardiomyocytes due to reactive oxidative stress. The study was conducted to compare the cardioprotective effects of carvedilol and $\alpha$-Tocopherol and to detect myocardial injury at early stage.

Methods: Cardiotoxicity was produced in a group of rabbits by single intravenous injection of doxorubicin; control group was treated with normal saline only. Third and fourth groups were pretreated with carvedilol $30 \mathrm{mg} / \mathrm{kg}$ bodyweight and $\alpha$-Tocopherol $200 \mathrm{mg} / \mathrm{kg}$ bodyweight respectively for ten days before injection of doxorubicin.

Results: Doxorubicin produced marked cardiotoxicity represented by raised levels of serum biomarkers (cTnI, LDH and CK-MB) and severe necrosis of cardiomyocytes on microscopic examination. Carvedilol and $\alpha$-tocopherol pretreatment resulted in decreased serum levels of biomarkers and improved the histological picture of heart tissue.

Conclusions: The outcome of doxorubicin chemotherapy can be made successful with the concurrent use of carvedilol or $\alpha$-tocopherol. Although carvedilol has more pronounced cardioprotective effects perhaps due to its antioxidant activity in addition to antiapoptotic, antiproliferative and antiinflammatory effects. Furthermore the quantitative cTnI estimation for detection of cardiotoxicity at early stage can lead to significant economic impact in management of cancer.
\end{abstract}

Keywords: Doxorubicin, Carvedilol, $\alpha$-tocopherol, Cardiac troponin I, LDH, CK-MB

\section{INTRODUCTION}

Doxorubicin is isolated from Streptomyces peuctius, a soil bacterium. ${ }^{1}$ Doxorubicin (Dox) is widely used for the treatment of several solid tumours and childhood malignancies since more than four decades. ${ }^{2}$ About $20 \%$ patients receiving doxorubicin may develop adverse cardiac effects. ${ }^{3}$

Its major anticancer effects occur through the inhibition of topoisomerase II $B$ and generation of DNA doublestrand breaks while cardiotoxicity is produced by virtue of its quinone group based metabolites that generate reactive oxygen species (ROS) \& free radicals. ${ }^{4-6}$ Cardiomyocytes are inherently more susceptible to oxidative stress. ${ }^{7}$ The free radicals \& ROS inflict mitochondrial and nuclear DNA lesions in cardiomyocytes. They disrupt mitochondrial bioenergetics and impaire expression of important cardiac proteins. ${ }^{8}$ Cardiomyocytes express nuclear (the $\beta$ isoform of Top2) and mitochondrial topoisomerases that are targeted by DOX. ${ }^{9,10}$ Doxorubicin metabolites also cause degradation of myofilaments and cytoskeletal proteins. ${ }^{11}$ These processes lead to cardiomyocyte death either by necrosis or by apoptosis, releasing the cardiospecific contractile proteins, cardiac troponins I (cTnI) and 
nonspecific cytosolic energy producing enzymes, creatine kinase MB (CK-MB) and lactate dehydrogenase (LDH) into the circulation. ${ }^{12-14} \mathrm{cTnI}$ is specifically expressed in myocardium and is 13 times more abundant than CK-MB in the myocardium. ${ }^{15}$ The quantitative estimation of the specific and sensitive biomarker cTnI determination detects the presence of cardiotoxicity at early subclinical stage that might prove to be helpful in cost effective management these of cases. ${ }^{16}$ The study will analyze the extent of protective role exerted by carvedilol and $\alpha$ lphatocopherol in prevention of cardiotoxic effects of DOX.

Several mechanisms have been proposed for the development of preventive strategies for DOX-induced cardiotoxicity. However, the protection conferred is not always effective on one hand and is expensive as well. ${ }^{17}$ The possible prevention by pretreatment with inexpensive $\alpha$-Tocopherol and carvedilol has produced noticeable decrease in cardiotoxicity and it may play a great role in adjuvant chemotherapy with doxorubicin. ${ }^{18,19}$

Carvedilol is a non-selective $\beta$ and $\alpha$ - adrenergic blocking agent with potent intrinsic anti-oxidant activity. It is an inexpensive free radical scavenger due to its hydroxylated metabolites and of carbazole moiety. It has antiapoptotic, antiproliferative and cardioprotective effects. It inhibits the apoptotic signaling in cardiomyocytes. It has marked protection in pathological processes associated with chemotherapeutic insults. ${ }^{20,21}$ Carvedilol reduces morbidity and mortality in patients with chronic heart failure and inhibits cardiac remodeling It prevents the depletion of endogenous antioxidants, such as vitamin $\mathrm{E}$ and glutathione and prevents mitochondrial dysfunction caused by doxorubicin. ${ }^{22,23}$ Carvedilol inhibits lipid peroxidation in myocardial cell membranes initiated by oxygen radicals. ${ }^{24}$

At high dosages, carvedilol prevents ventricular tachycardia in heart failure associated with chemotherapy. ${ }^{25}$ In addition to its antioxidant properties, carvedilol has been reported to have an anti-inflammatory effect by inhibiting interleukons (IL-10 and IL-18) in patients with cardiac injury and IL- 8 via NF- $\kappa$ B in the human endothelial cells involved in myocardial cell death. $^{26}$

Alpha-tocopherol is a lipid-soluble, inexpensive organic compound thought to prevent the propagation of free radical damage in biological membranes. ${ }^{27}$ It inhibits lipid peroxidation in the presence of endogenous vitamin $\mathrm{C}$ and selenium in cytosol. ${ }^{28,29}$

Various studies in preclinical and clinical models showed the effectiveness of $\alpha$-tocopherol in mitigating cardiac toxicity of chemotherapeutic agents like doxorubicin where oxidative stress plays a major role. ${ }^{30}$ It can prevent the pro-inflammatory cytokines and chemokines induced by doxorubicin. ${ }^{31}$

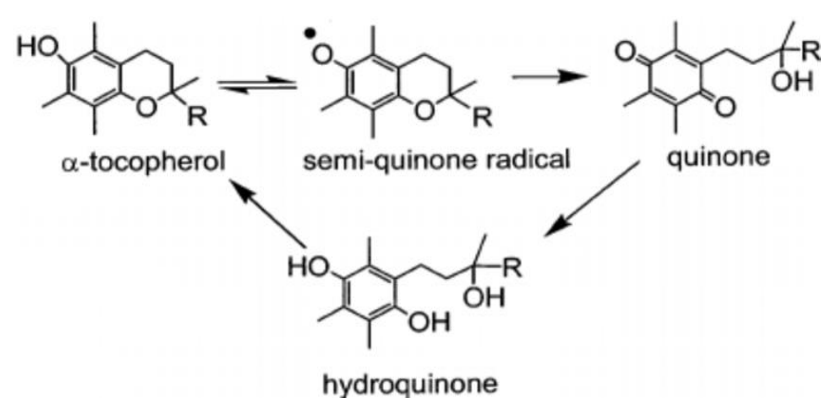

Figure 1: Interaction of $\alpha$ - tocopherol with $\mathbf{R}$-(free) radical.

\section{METHODS}

The in-vivo lab based randomized controlled study was carried out on twenty-four adult healthy male rabbits weighing 1.0 to $1.5 \mathrm{~kg}$. This study was conducted from August to December 2018. The rabbits were acclimatized with the environment of animal house keeping at standard normal conditions and fed with fresh green fodder, cereals and tap water ad libitum.

Pharmaceutical brands of doxorubicin HCL from ParkDavis and carvedilol were procured from Ferozsons Pak Ltd. $\alpha$-tocopherol, CK-MB and LDH commercial kit were purchased from Merck Pharma while cTnI Beckman Coulter kit from PMA.

The rabbits were randomly divided into four groups, Group A: $(n=6)$ Kept as control receiving 0.9\% sodium chloride $(\mathrm{NaCl})$ solution $2 \mathrm{ml}$ daily by gavage for 11 days. Group B: $(n=6)$ was administered doxorubicin injection $12 \mathrm{mg} \mathrm{kg}^{-1}$ body weight (BW) into marginal ear vein on $10^{\text {th }}$ day of study. Group $C:(n=6)$ was given carvidelol $30 \mathrm{mg} \mathrm{kg}^{-1}$ bodyweight and Group D: $(\mathrm{n}=6) \alpha-$ tocopherol $200 \mathrm{mg} \mathrm{kg}^{-1}$ of body weight by gavage from day 1 to day 11 plus doxorubicin $12 \mathrm{mg} \mathrm{kg}^{-1}$ body weight IV in single dose on $10^{\text {th }}$ day of study.

Estimation of cTnI, CK-MB and LDH by specific kit methods was performed on blood drawn at the beginning of the study and on the final day after twenty-four hours of doxorubicin administration. The blood was allowed to clot at room temperature and centrifuged at $3000 \mathrm{rpm}$ for 15 minutes. Serum separated was stored in clean, dry serum storage vials at $-20^{\circ} \mathrm{C}$ for analysis of biomarkers.

cTnI was detected by Immunoassay systems of Beckman Coulter kit (Access Accu TnI) on (Access 2) made in USA. A cut off value of $0.50 \mathrm{ng} / \mathrm{ml}$ cTnI was recommended for diagnosis of necrosis. CK-MB estimation was done by optimized IFCC method and lactate dehydrogenase (LDH) was measured by UV kinetic method using commercial kit from Merck.

Rabbits were weighed and then sacrificed to take out the heart. Heart tissue sections were prepared for examination. Histological sections of the myocardium of 
all the rabbits were assessed qualitatively and quantitatively. Qualitatively, doxorubicin (DOX) induced cardiac damage was recognized by the presence of marked interstitial edema, inflammation, perinuclear vacuolization, disorganization and degeneration of the myocardial fibrils. Semiquatitative morphological grading was done by using Billingham scoring method.

Statistical results of serum analysis were expressed as means \pm standard error of means (SEM) calculated by using One Way ANOVAs on SPSS 22. The results of histopathology were analyzed by using the Chi square test. The difference between two observations was considered as significant if the $\mathrm{p}$ value was found $<0.05$.

\section{RESULTS}

\section{Observation of individual parameters}

\section{Body weight}

Mean body weight of Gp-A (treated with normal saline) increased by $12.52 \pm 2.69$ percent of the initial weight while it fell by $26.88 \pm 2.03$ percent in Gp-B (treated with $12 \mathrm{mg} / \mathrm{kg}$ of doxorubicin). The animals of $\mathrm{Gp}-\mathrm{C}$ (treated with $12 \mathrm{mg} / \mathrm{kg}$ of doxorubicin plus carvedilol $30 \mathrm{mg} / \mathrm{kg}$ ) and Gp-D treated with $12 \mathrm{mg} / \mathrm{kg}$ of doxorubicin plus Alpha tocopherol $200 \mathrm{mg} / \mathrm{kg}$ ) showed less weight loss of $5.55 \pm 7.78$ and $4.57 \pm 2.48$ percent as compare to Gp-B.

\section{Serum biomarkers}

\section{Serum cardiac troponin I (cTnI)}

Cardiac troponin I levels remained well below normal over the whole period of study i.e. within the range of $0.03 \pm 0.00 \mathrm{ng} / \mathrm{l}$ in $\mathrm{Gp}-\mathrm{A}$ but there was marked rise of cTnI upto $10.55 \pm 0.00 \mathrm{ng} / \mathrm{l}$ in $\mathrm{Gp}-\mathrm{B}$ with statistically significant difference $(\mathrm{p}<0.05)$ as compared to Gp-A. In Gp-C cTnI levels $(2.44 \pm 1.00 \mathrm{ng} / \mathrm{l})$ were high as compared to $\mathrm{Gp}-\mathrm{A}$ but much low as compared to Gp-B with statistically significant difference $(\mathrm{p}<0.05)$. Similarly in Gp-D cTnI levels $(2.85 \pm 1.00 \mathrm{ng} / \mathrm{l})$ were high as compared to Gp-A but much low as compared to Gp-B with statistically significant difference $(\mathrm{p}<0.05)$ (Table 1$)$.

\section{Serum $C K-M B$}

Serum CK-MB increased significantly to $346 \mathrm{U} / \mathrm{L} \pm 37$ in Gp-B as compared with control Gp-A $129 \pm 4$ U/l. P value was found to be statistically significant $(\mathrm{p}<0.05)$, (Table 1). Again Gp-C showed less increase (186 U/L \pm 10.00$)$ of CK-MB as compared to Gp-B and value of $\mathrm{P}$ was statistically significant $(\mathrm{p}<0.05), \quad($ Table 1$)$. Gp-D receiving $\alpha$-tocopherol $200 \mathrm{mg} / \mathrm{kg}$ also showed decreased levels (186 U/L) of CK-MB as compared to Gp-B and value of $P$ was statistically significant $(p<0.05)$. When value of Gp-D CK-MB was compared with vaue of Gp-C it was not found significant (Table 1).

Table 1: Serum biomarkers of rabbits in Gp A, with normal saline (n=6), Gp B with doxorubicin (n=6), Gp C and Gp D pretreated with carvedilol with $\alpha$-tocopherol before receiving doxorubicin.

\begin{tabular}{|c|c|c|c|c|c|c|c|c|c|}
\hline \multirow{2}{*}{ Serum tests } & \multicolumn{4}{|l|}{ Day 0} & \multicolumn{5}{|c|}{ Day 11} \\
\hline & Gp A & Gp B & Gp C & Gp D & Gp A & Gp B & Gp C & Gp D & $P$ value \\
\hline LDH (U/I) & 722 & 643 & 799 & 698 & 760 & 1421 & 630 & 646 & \\
\hline SEM \pm & 25 & 10 & 36 & 11 & 26 & 114 & 25 & 55 & $0.000 *$ \\
\hline CKMB (U/I) & 119 & 122 & 133 & 119 & 129 & 346 & 197 & 186 & \\
\hline SEM \pm & 10 & 15 & 10 & 6 & 4 & 37 & 10 & 7 & $0.000^{*}$ \\
\hline cTnI (ng/l) & 0.03 & 0.03 & 0.03 & 0.03 & 0.03 & 10.5 & 2.44 & 2.85 & \\
\hline SEM \pm & 0 & 0 & 0 & 1 & 0 & 0 & 1 & 1 & $0.000 *$ \\
\hline
\end{tabular}

\section{Serum $L D H$}

Serum LDH raised markedly i.e. up to $1421 \pm 114 \mathrm{U} / 1$ in $\mathrm{Gp}-\mathrm{B}$ as compared to $\mathrm{Gp}-\mathrm{A}$ and value of $\mathrm{p}<0.005$ significant (Table 1). Gp-C depicted no rise in value of LDH $(636 \pm 55 \mathrm{U} / \mathrm{l})$. On comparison with $\mathrm{Gp}-\mathrm{B}$, value of $\mathrm{p}$ was significant (0.000). In Gp-D value of LDH was 646 $\mathrm{U} / \mathrm{l}$. On comparison with $\mathrm{Gp}-\mathrm{B}, \mathrm{p}$ value was found significant (Table 1).

\section{Histological examination}

Histological examination of section of rabbit hearts from the Gp-A (control group) showed normal morphology with no damage to the cardiac tissue ( 0 grade necrosis) Figure 2A.
Microscopic examination of Gp-B (toxic group) revealed signs of massive necrosis. None of them were normal. Among total rabbit hearts $83.3 \%$ showed grade 3 necrosis and $16.7 \%$ of grade 2 necrosis. The sections of free wall of ventricles showed marked interstitial oedema, infiltration with inflammatory cells, vacuolization and nuclear material clumping. In some of the sections there was neutrophil infiltration of muscle fibres with loss of myofibril arrangement (Figure 2B).

In $\mathrm{Gp}-\mathrm{C}$, (treated with carvedilol and doxorubicin), $66.7 \%$ of heart section revealed mild necrosis of grade 1 only and complete prevention was exhibited by $16.7 \%$ and similar number showed moderate toxicity (Figure 3C). In Gp-D, heart sections showed less damage. Statistically only $16.7 \%$ revealed severe necrosis, 66.7 
percent of sections were with moderate grade of 3 necrosis and $16.7 \%$ mild changes in morphology exhibiting grade 2 Figure 2D.
When chi square was applied to all the groups, it showed significant difference among the groups. The value of $\mathrm{p}$ is 0.009 (Table 2).
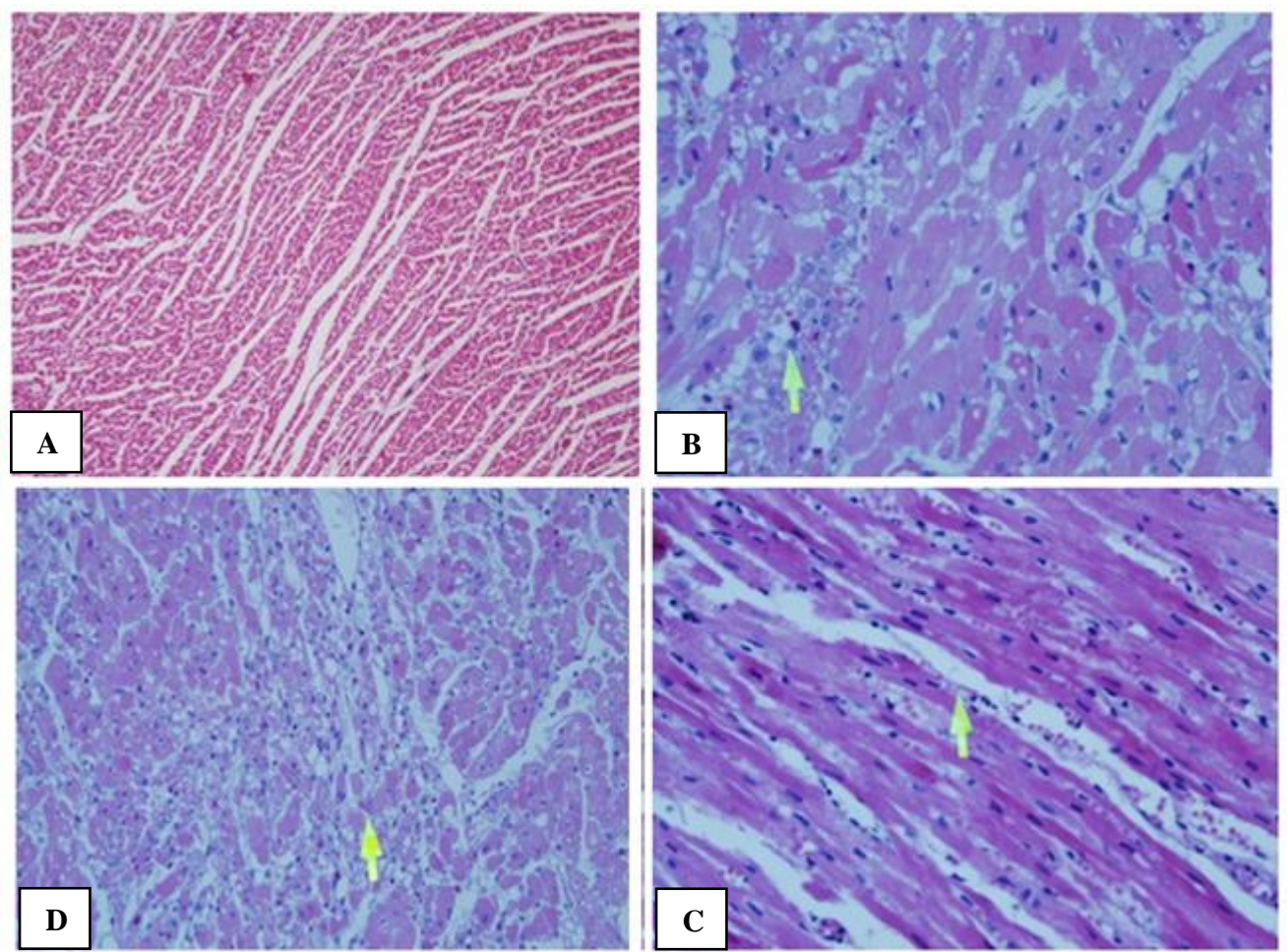

Figure 2: Clockwise from top left, (A) microscopic picture of (H\&E)-stained biopsy specimens of rabbit cardiomyocytes from normal (Gp-A) showing normal architecture (300X); (B) micrograph of rabbit cardiomyocytes treated with $12 \mathrm{mg} / \mathrm{kg}$ of doxorubicin showing marked degree of vacuolization and disrupted myofibiril arrangement and swollen nuclei and infiltration with inflammatory cells (300X); (C) micrograph of rabbit cardiomyocytes after treatment with doxorubicin $12 \mathrm{mg} / \mathrm{kg}$ plus carvedilol $30 \mathrm{mg} / \mathrm{kg}(300 X)$ showing less degree of damage as compared to Gp-B as well (D); (D) micrograph of rabbit cardiomyocytes after treatment with doxorubicin $12 \mathrm{mg} / \mathrm{kg}$ plus $\alpha$-tocophrol $200 \mathrm{mg} / \mathrm{kg}(300 \mathrm{X})$ showing moderate degree of damage.

Table 2: Histological grades.

\begin{tabular}{|c|c|c|c|c|c|c|}
\hline & & & Grou & & & Total \\
\hline & & & $\mathrm{B}$ & $\mathrm{C}$ & D & Lual \\
\hline & Normol & Count & 0 & 1 & 0 & 1 \\
\hline & Normal & $\%$ within groups & 0.0 & 16.7 & 0.0 & 5.6 \\
\hline & Mild & count & 0 & 4 & 1 & 5 \\
\hline Grades & 10mu & $\%$ within groups & 0.0 & 66.7 & 16.7 & 27.8 \\
\hline Grades & Moderate & count & 1 & 1 & 4 & 6 \\
\hline & vioderate & $\%$ within groups & 16.7 & 16.7 & 66.7 & 33.3 \\
\hline & & count & 5 & 0 & 1 & 6 \\
\hline & Severe & $\%$ within groups & 83.3 & 0.0 & 16.7 & 33.3 \\
\hline Total & & count & 6 & 6 & 6 & 18 \\
\hline 10tal & & $\%$ within groups & 100.0 & 100.0 & 100.0 & 100.0 \\
\hline Chi-squ & & & & & & \\
\hline & & Value & & & Asymp. & ided) \\
\hline Pearson & are & $17.200^{\mathrm{a}}$ & & & 0.009 & \\
\hline Likeliho & & 18.729 & & & 0.005 & \\
\hline Linear-l & association & 2.370 & & & 0.124 & \\
\hline $\mathbf{N}$ of vali & & 18 & & & & \\
\hline
\end{tabular}




\section{DISCUSSION}

This study was aimed to compare the cardioprotective potentials of carvedilol and $\alpha$-tocopherol in doxorubicin induced cardiotoxicity in rabbits.

In our study, group of animals (Gp-B) receiving doxorubicin in single toxic dose showed markedly deranged serum biomarkers of cardiac injury, with significant difference in serum $\mathrm{LDH}$ and increase of $59.32 \%$, serum CK-MB with increase of $146.49 \%$ and serum cTnI values with increase of $33210.53 \%$ as compared to control group of animals (Gp-A). The microscopic examination of heart sections of Gp-B revealed grade 4 necrosis in most of the tissue while the histological picture of Gp-A animal was completely normal

Simunek, Che and his colleagues observed similar changes both in morphology and biomarkers following the use of doxorubicin in rabbits. ${ }^{32,33}$ Sawyer and his colleagues in preclinical studies both in vitro and in vivo and Cardinale and colleagues in clinical study on cancer patients established that doxorubicin caused a significant and dose-dependent cardiomyocyte apoptosis and myocytes death at 24-48 $\mathrm{h}$ after the injection as seen in our histological reports. ${ }^{14,15}$ Furthermore, many other researchers had documented the elevated concentrations of serum biomarkers troponin I, CK-MB and LDH associated with doxorubicin-induced cardiomyocytes death and our results were found consistent with them.

Doxorubicin is front-line therapy to treat a large number of different malignancies including ever increasing breast cancer and a significant fraction (20\%) of patients experience drug-induced cardiotoxicity leading to longterm morbidity or mortality and this clinical impact is growing with increasing cancer survivorship. ${ }^{11,34}$ In our study the rationale behind the measurement of LDH and CK-MB was their historical value and our emphasis remained on cTnI as more specific and sensitive biomarker as cTnI is considered gold standard biomarker of cardiac injury in all mammalian species and this was acknowledged by The Food and Drug Administration (FDA) and European Medicines Agency (EMA) to detect the early anthracyclin-induced cardiotoxocity. ${ }^{15}$

The second part consists of comparative study the cardioprotection provided by carvedilol and $\alpha$-tocopherol. The $\mathrm{Gp}-\mathrm{C}$ animals pretreated with $30 \mathrm{mg} / \mathrm{kg}$ carvedilol for 10 days before the exposure of doxorubicin revealed the levels of cTnI showing significant difference with $76.87 \%$ less increase, CK-MB with $42.93 \%$ less increase and $\mathrm{LDH}$ with $57.42 \%$ less increase in $\mathrm{Gp}-\mathrm{C}$ as compared to Gp-B. Histological changes were also comparable with significant difference $(\mathrm{p}<0.000)$. The histological picture shifted from grade 3 necrosis to only of grade 1 while one-forth sections exhibited quite normal architecture of myofibrils. The weight loss $(4.57 \pm 2.48 \%)$ among the animals of this group was of lesser degree.
These findings are in consistent with in vivo research study carried out by Hadi and his co-workers and Arozal et al who studied the cardioprotective effects of carvedilol after cumulative dose of doxorubicin in rats. ${ }^{35,36}$

Increased oxidative stress, nuclear DNA lesions, mitochondrial dysfunctions, interference in signalling pathway and apoptosis have been implicated in the cardiotoxicity of doxorubicin. Carvedilol is a third generation non-selective $\beta$-adrenergic blocking agent with potent intrinsic anti-oxidant activity. Carvedilol is a highly lipophilic compound and attains much higher concentration in cytoplasm and mitochondria. Carvedilol and several of its metabolites prevent lipid peroxidation caused by doxorubicin. The antioxidant properties of carvedilol result from the carbazole moiety in its structure. Carvedilol is a potent antioxidant, 10 fold more potent than vitamin E. Its metabolites are 30-80 times more potent than carvedilol and up to 1000-fold more potent than vitamin $\mathrm{E}^{37}$

Gp-D animals received $\alpha$-tocopherol $200 \mathrm{mg} / \mathrm{kg} 10$ days before the exposure of doxorubicin. The results of LDH showing significant difference with $27.60 \%$ less increase, CK-MB with $46.31 \%$ less increase and cTnI with $73.01 \%$ less increase when compared to Gp-B. Histological changes were also comparable with $(\mathrm{p}<0.000)$. Similar findings were observed by Hadi et al in vivo research study in which rats were exposed to intraperitoneal doxorubicin after pre-treatment with $\alpha$-tocopherol. Few other studies in rabbit performed in the past showed the effectiveness of $\alpha$-tocopherol in mitigating cardiac toxicity of acute high doxorubicin doses. ${ }^{35}$ In another rabbit model with chronic Dox-induced toxicity, $\alpha$ tocopherol exerted protective effect when was administrated in high dosage as a pre-treatment or given in combination with vitamin $\mathrm{A}$, reducing myocardial damage. ${ }^{38}$

\section{CONCLUSION}

In conclusion the results of this study revealed that DOX induced cardiotoxicity can be recognized at earlier stage by quantitative estimation of cTnI for appropriate intervention. Troponin negative subjects can be excluded from cardiac monitoring programs. Similarly pretreatment with carvedilol and $\alpha$-tocopherol attenuated the doxorubicin induced cardiotoxicity. Among the two antioxidants, carvedilol has more pronounced cardioprotective effects. Perhaps carvedilol has more than antioxidant activity as it has antiapoptotic, antiproliferative and anti-inflammatory effects rendering it more cardioprotective. Further exploratory work needs to be done to determine the exact mechanisms in amelioration of DOX induced cardiotoxicity in animal and human models.

\section{ACKNOWLEDGEMENTS}

This work was partly supported by grant from National University of Sciences and Technology (NUST), Islamabad, Pakistan. 
Funding: No funding sources

Conflict of interest: None declared

Ethical approval: The study was approved by the Institutional Ethics Committee

\section{REFERENCES}

1. Schulz D, Nachtigall J, Geisen U, Kalthoff H, Imhoff JF, Fiedler HP, Süssmuth RD. Aranciamycin anhydride, a new anthracycline-type antibiotic isolated from Streptomyces. J Antibiot. 2010;63(7):397-9.

2. Thorn CF, Oshiro C, Marsh S, Hernandez-Boussard $\mathrm{T}$, McLeod H, Klein TE, et al. Doxorubicin pathways:pharmacodynamics and adverse effects. Pharmacogenetics Genomics. 2010;21(7):440-6.

3. Mulrooney DA, Yeazel MW, Kawashima T, Mertens AC, Mitby P, Stovall M, et al. Cardiac outcomes in a cohort of adult survivors of childhood and adolescent cancer:retrospective analysis of the Childhood Cancer Survivor Study cohort. $\mathrm{Br}$ Med J. 2009;339:b4606.

4. Minotti G, Menna P, Salvatorelli E, Cairo G, Gianni L. Anthracyclines: molecular advances and pharmacologic developments in antitumor activity and Cardiotoxicity. Pharmacol Rev. 2004;56:185229.

5. Matt S, Hofmann TG. The DNA damage-induced cell death response:a roadmap to kill cancer cells. Cellular Molecular Life Sci. 2016;73(15):2829-50.

6. L'Ecuyer T, Sanjeev S, Thomas R, Novak R, Das L, Campbell W, et al. DNA damage is an early event in doxorubicin-induced cardiac myocyte death. Am J Physiol. 2006;291(3):1273-80.

7. McGowan JV, Chung R, Maulik A, Piotrowska I., Walker JM, Yellon DM. Anthracycline chemotherapy and cardiotoxicity. Cardiovasc Drugs Thera. 2017;31(1):63-75.

8. Kankeu C, Clarke K, Passante E, Huber HJ. Doxorubicin-induced chronic dilated cardiomyopathy the apoptosis hypothesis revisited. J Mol Med. 2016;95(3):239-48.

9. Olson RD, Gambliel HA, Shadle SE, Cusack BJ. Doxorubicin cardiac dysfunction. Cardiovasc Toxicol. 2005;5(3):269-83.

10. Zhang S, Liu X, Bawa-Khalfe T, Lu LS, Lyu YL, Liu $\mathrm{LF}$, et al. Identification of the molecular basis of doxorubicin-induced cardiotoxicity. Nat Med. 2012;18(11):1639-42.

11. Cappetta D, Angelis AD, Sapio L, Prezioso L, Illiano M, Quaini F, et al. Oxidative Stress and Cellular Response to Doxorubicin:A Common Factor in the Complex Milieu of Anthracycline Cardiotoxicity Oxid Med Cell Longev. 2017;2017:1521020.

12. Ghigo A, Li M, Hirsch E. New signal transduction paradigms in anthracycline-induced cardiotoxicity. Biochim Biophys Acta. 2016;1863(7):1916-25.

13. Henriksen PA. Anthracycline cardiotoxicity: An update on mechanisms, monitoring and prevention. Heart. 2018;104:971-7.
14. Sawyer DB, Zuppinger C, Miller TA, Eppenberger HM, Suter TM. Modulation of anthracycline-induced myofibrillar disarray in rat ventricular myocytes by neuregulin- $1 \beta$ and anti-erbB2:potential mechanism for trastuzumab-induced cardiotoxicity. Circulation. 2010;105:1551-4.

15. Cardinale D, Sandri MT. Role of biomarkers in chemotherapy-induced cardiotoxicity. Prog Cardiovasc Dis. 2010;53(2):121-9.

16. Sawaya H, Sebag IA, Plana JC. Early detection and prediction of cardiotoxicity in chemotherapy-treated patients. Am J Cardiol. 2011;107:1375-80.

17. Gianni L, Herman EH, Lipshultz SE, Minotti G, Sarvazyan N, Sawyer DB. Anthracycline cardiotoxicity:from bench to bedside. J Clin Oncol. 2008;26(22):3777-84.

18. Kalam K, Marwick HT. Role of cardioprotective therapy for prevention of cardiotoxicity with chemotherapy: A systematic review and metaanalysis. Euro J Cancer. 2013;49(13):2900-9.

19. Farías JG, Molina VM, Carrasco RA, Zepeda AB, Figueroa E, Letelier P, et al. Antioxidant therapeutic strategies for cardiovascular conditions associated with oxidative stress. Nutrients. 2017;1(9):966.

20. Jhorawat R, Kumari S, Varma SC, Rohit MK, Narula $\mathrm{N}$, Suri V, et al. Preventive role of carvedilol in adriamycin-induced cardiomyopathy. Indian $\mathrm{J}$ Med Res. 2016;144(5):725-9.

21. Zhou Q, Xiao J, Jiang D, Wang R, Vembaiyan K, Wang A, et al. Carvedilol and its new analogs suppress arrhythmogenic store overload-induced Ca2+ release. Nat Med. 2011;17(8):1003-9.

22. Watanabe K, Arozal W, Sari FR, Arumugam S, Thandavarayan AR, Suzuki K, et al. The Role of Carvedilol in the Treatment of Dilated and Anthracyclines-Induced Cardiomyopathy. Kufa Med J. 2011;15(1):314.

23. Rehsia NS, Dhalla NS. Mechanisms of the beneficial effects of beta-adrenoceptor antagonists in congestive heart failure. Exp Clin Cardiol. 2010;15(4):86-95.

24. Sari FR, Arozal W, Watanabe K, Harima M, Veeravedu PT, et al. Carvedilol Attenuates Inflammatory-Mediated Cardiotoxicity in Daunorubicin-Induced Rats. Pharmaceut 2011;4(3):551-66.

25. Sharma A, Herzo C. Beneficial effects of Carvedilol in dilated cardiomyopathy in dialysis patients. J Am Coll Cardiol. 2012;59(13):887.

26. Oliveira PJ, Bjork JA, Santos MS. Carvedilolmediated antioxidant protection against doxorubicininduced cardiac mitochondrial toxicity. Toxicol Appl Pharmacol. 2004;200:159-68.

27. Brigelius-Flohé R, Galli F. Vitamin E:A vitamin still awaiting the detection of its biological function. Mol. Nutr. Food Res. 2010;54:583-8.

28. Ajmal K, Khan BT, Hashim R, Bakhtiar B. Doxorubicin-Induced Cardiotoxocity Detection. Pak Armed Forces Med J. 2014;64(3):384-90.

29. Atkinson J, Harroun T, Wassall SR, Stillwell W, Katsaras J. The location and behaviour of -tocopherol 
in membranes. Mol Nutr Food Res. 2010;54(5):64151.

30. Zinng JM. Molecular and cellular activities of vitamin E analogues. Mini Rev Med Chem. 2014;7(5):543-58.

31. Jiang Q. Natural Forms of Vitamin E as Effective Agents for Cancer Prevention and Therapy. Advac in Nutri. 2017;8(6):850-67.

32. Simunek T, Sterba M, Popelova O, Adamcova M, Hrdina R, Gesi V. Anthracycline-induced cardiotoxicity: overview of studies examining the roles of oxidative stress and free cellular iron. Pharmacolo Repo. 2009;61(1):154-71.

33. Che FF, Liu Y, Xu CG. Schisandrin B prevents doxorubicin-induced cardiotoxicity in rabbits. Sichuan Da Xue Xue Bao Yi Xue Ban. 2010;41(1):24-8.

34. Howlader N, Noone AM, Krapcho M, Miller D, Brest A, Yu M, et al. SEER Cancer Statistics Review, 1975-2016. National Cancer Institute, Bethesda, MD. 2017.

35. Hadi N, Yousif GN, Al-amran GF, Huntei NK, Mohammad BI, Ali SJ. Vitamin E and telmisartan attenuates doxorubicin induced cardiac injury in rat through down regulation of inflammatory response. BMC Cardiovasc Disorders. 2012;12:63.

36. Arozal W, Sari RF, Watanabe K, Arumugam S, Thandavarayan AR, Suzuki K. Carvedilol Attenuates Inflammatory-Mediated Cardiotoxicity in Daunorubicin Induced Rats. Pharmaceuticals. 2011;4(3):551-66.

37. Cochera F, Dinca D, Bordejevic DA, Citu IM, Mavrea AM, Andor M, et al. Nebivolol effect on doxorubicin-induced cardiotoxicity in breast cancer. Cancer Management Res. 2018;10:2071-81.

38. Abbas A, Hadi A, Saeed CA, Ali C, Reza T, Sadaf D, Soheila R. Protective effects of omega-3, atorvastatin, vitamin $\mathrm{E}$ and vitamin $\mathrm{C}$ against doxorubicin-induced cardiotoxicity in rats: a comparison study. Physiol Pharmacol. 2018;22:6372 .

Cite this article as: Ajmal K, Ajmal M, Afzal A, Saima, Naeem U, Sikandar A. Comparison of protective effects of carvedilol and $\alpha$-tocopherol on doxorubicin-induced cardiotoxocity. Int J Basic Clin Pharmacol 2019;8:1944-50. 